\title{
Retrospective immunohistological study of autopsied lungs in patients with acute exacerbation of interstitial pneumonia managed with extracorporeal membrane oxygenation
}

\author{
Yoshiko Kida $^{1}$, Shinichiro Ohshimo ${ }^{1}$, Michihito Kyo ${ }^{1}$, Koji Hosokawa ${ }^{1}$, Vishwa Jeet Amatya ${ }^{2}$, \\ Yukio Takeshima ${ }^{2}$, Nobuaki Shime ${ }^{1}$ \\ ${ }^{1}$ Department of Emergency and Critical Care Medicine, ${ }^{2}$ Department of Pathology, Graduate School of Biomedical and Health Sciences, Hiroshima \\ University, Hiroshima, Japan \\ Contributions: (I) Conception and design: None; (II) Administrative support: None; (III) Provision of study materials or patients: None; (IV) \\ Collection and assembly of data: None; (V) Data analysis and interpretation: K Hosokawa, M Kyo, S Ohshimo, N Shime, VJ Amatya, Y Takeshima; \\ (VI) Manuscript writing: All authors; (VII) Final approval of manuscript: All authors. \\ Correspondence to: Yoshiko Kida, MD, PhD. Department of Emergency and Critical Care Medicine, Graduate School of Biomedical and Health \\ Sciences, Hiroshima University, 1-2-3 Kasumi, Minami-ku, Hiroshima 734-8551, Japan. Email: kida@hiroshima-u.ac.jp.
}

Background: Acute exacerbation of interstitial pneumonia (AE-IP) is a life-threatening pulmonary condition that involves various pathogeneses. In patients with AE-IP who need mechanical ventilation with high driving pressure and oxygen concentration, veno-venous extracorporeal membrane oxygenation ( $\mathrm{V}-\mathrm{V}$ ECMO) may diminish alveolar epithelial damage by decreasing ventilator settings. The pathophysiological benefit of this therapeutic option is not well investigated.

Methods: We retrospectively collected 15 autopsied patients with AE-IP who were treated with mechanical ventilation in the intensive care unit (ICU) at Hiroshima University Hospital (Hiroshima, Japan) between 2010 and 2016. The patients were grouped by whether they were managed with mechanical ventilation only (the ventilator group, $\mathrm{n}=6$ ) or with mechanical ventilation and V-V ECMO (the ECMO group, $\mathrm{n}=9$ ).

Results: The median age of the ventilator and ECMO group patients were similar (65 and 64 years, respectively). The severity score APACHE II in the ECMO group (35.0) is significantly higher than that of ventilator group (14.5) $(\mathrm{P}=0.006)$. Ventilator days were significantly shorter in the ventilator group (17.5 days) than in the ECMO group (30.0 days) ( $\mathrm{P}=0.04)$. Compared with the ECMO group, the ventilator group had a stronger Masson-trichrome stain grade (4 vs. 6, $\mathrm{P}=0.04)$ and higher immunoreactivity grades for Krebs von den Lungen-6 (4 vs. 6, P=0.04) and IL-8 (3 vs. 6, P=0.02). Between the ventilator and ECMO groups, the immunoreactivity grades of angiopoietin 2 ( 4 vs. 1, $\mathrm{P}=0.08)$ and receptor for advanced glycation end products ( 2 vs. $1, \mathrm{P}=0.52$ ) did not differ.

Conclusions: The lungs of mechanically ventilated AE-IP patients treated with V-V ECMO had decreased fibrosis, endothelial injury, and inflammation. This finding suggests the lung-protective efficacy of adjunctive $\mathrm{V}-\mathrm{V}$ ECMO therapy.

Keywords: Acute respiratory syndrome; lung protection; biomarker

Submitted Jul 28, 2019. Accepted for publication Oct 17, 2019.

doi: $10.21037 /$ jtd.2019.11.09

View this article at: http://dx.doi.org/10.21037/jtd.2019.11.09

(C) Journal of Thoracic Disease. All rights reserved. 


\section{Introduction}

Acute exacerbation of interstitial pneumonia (AE-IP) is a lifethreatening condition characterized by acute worsening of dyspnea and progressive bilateral radiographic infiltrates (1-3). Pharmacological interventions such as corticosteroids or immunosuppressants are limited (4,5). These patients require prolonged mechanical ventilation as supportive care (6). However, high driving pressures and oxygen concentrations are usually needed during ventilation care because of low lung compliance and oxygenation capacity, which are highly associated with worsening lung injury and poor outcome $(6,7)$.

Veno-venous extracorporeal membrane oxygenation ( $\mathrm{V}-\mathrm{V}$ ECMO) is an emerging tool for patients with acute hypoxemic respiratory failure (8). Damaged lungs are particularly vulnerable to the mechanical stress of ventilation (9-11); therefore, respiratory management using ECMO may reduce alveolar epithelial damage and thereby improve the survival rate (12). In addition, serum, epithelial, endothelial, and inflammatory biomarkers are associated with patients with acute respiratory distress syndrome (ARDS) (13). Some pathological processes and findings of the diffuse alveolar damage pattern are similar between ARDS and AE-IP (14-18).

Thus, we hypothesize that lung protective management using $\mathrm{V}-\mathrm{V}$ ECMO could aid in minimizing alveolar epithelial injury and the resultant fibrosis in patients with AE-IP, and serum, epithelial, endothelial, and inflammatory biomarkers, that are relevant for ARDS (13), of AE-IP patients are decreased if $\mathrm{V}-\mathrm{V}$ ECMO reduce the risk of VILI. However, histopathological evaluations of the lungs in patients with AE-IP who undergo V-V ECMO are not well investigated. The aim of the study was to compare the pathological changes in the expressions of various biomarkers in the autopsied lungs of patients with AE-IP who were managed with and without V-V ECMO.

\section{Methods}

\section{Study patients}

The total number of AE-IP patients who were admitted in the ICU at the Hiroshima University Hospital between January, 2010 and December, 2016 was eighty-seven patients. We retrospectively collected lung tissue specimens from consecutive patients who underwent autopsy $(n=15)$ and who were diagnosed with AE-IP and were treated with mechanical ventilation. Lung specimens were sampled within 48 hours after the patients' death. Patients' were divided into two groups: (I) patients who were managed with mechanical ventilation alone or with noninvasive positive pressure ventilation (the ventilator group, $n=6$ ) and (II) patients who were managed with mechanical ventilation plus V-V ECMO (the ECMO group, n=9). The patients' characteristics were retrospectively extracted from the medical records, and included age, sex, serum Krebs von den Lungen-6 (KL-6) value, length of intensive care unit (ICU) stay, duration of mechanical ventilation, and duration of $\mathrm{V}-\mathrm{V}$ ECMO. The experiments in this study comply with the current laws of Japan, and were approved by the ethics committee in our institution (Hiroshima University, Hiroshima, Japan; project approval No.: RIN-231).

\section{Definition and diagnosis of AE-IP}

The diagnosis of AE-IP was determined, based on the current recommendations, as follows (19): (I) worsening dyspnea within days to weeks; (II) abnormal gas exchange, as defined by a low partial pressure of arterial oxygen/ fraction of inspired oxygen $\left(\mathrm{PaO}_{2} / \mathrm{F}_{1} \mathrm{O}_{2}\right)$ ratio or a decrease in $\mathrm{PaO}_{2}$; (III) new radiographic opacities such as groundgrass areas, irregular linear opacities, and honeycombing without any signs suggestive of bacterial pneumonia, left heart failure, or pulmonary embolism (20,21); and (IV) elevated serum levels of fibrosis biomarkers such as lactate dehydrogenase, pulmonary surfactant protein D (SP-D), and KL-6.

\section{Mechanical ventilation and ECMO management}

For all patients in the ventilator group, the ventilation mode was pressure-controlled assist-control ventilation, airway pressure release ventilation, or high-frequency oscillatory ventilation. The decision to initiate mechanical ventilation was at the discretion of the attending physician. The $\mathrm{PaO}_{2}$ and $\mathrm{PaCO}_{2}$ were maintained to approximately $60-70$ and $40-55 \mathrm{mmHg}$, respectively, with a limiting plateau-pressure of $<40 \mathrm{cmH}_{2} \mathrm{O}$, while avoiding hyperoxemia (i.e., $\mathrm{SpO}_{2}$ $<96 \%$ or $\mathrm{PaO}_{2}<80 \mathrm{mmHg}$ ).

The criteria for initiating $\mathrm{V}-\mathrm{V}$ ECMO to treat AEIP were a $\mathrm{PaO}_{2} / \mathrm{F}_{\mathrm{I}} \mathrm{O}_{2}$ ratio $<100, \mathrm{pH}<7.2$, and/or severe respiratory distress (e.g., tachypnea). The exclusion criterion was an age of 70 years or above.

The V-V ECMO treatment was established within at least 7 days from starting mechanical ventilation, and as early as possible after meeting the criteria. The ECMO 
flow was set at 3.5-4.0 L/min with a targeted $\mathrm{SpO}_{2}$ of $85 \%-90 \%$. For all patients managed with ECMO, unfractionated heparin was used for anticoagulation with a targeted activated partial thromboplastin time of 1.5-2.5 times normal. Patients were sedated with midazolam, dexmedetomidine, and/or fentanyl. Spontaneous breathing was mostly inhibited. In our institution, initiating $\mathrm{V}-\mathrm{V}$ ECMO in patients is attempted immediately after patients meet the criteria for ECMO to avoid causing further alveolar damage due to prolonged high-pressure settings of mechanical ventilation. The setting of the ventilator in the ECMO group was as follows: $\mathrm{F}_{\mathrm{I}} \mathrm{O}_{2}$ of approximately 0.21 and high positive end-expiratory pressure and peak pressure $<30 \mathrm{cmH}_{2} \mathrm{O}$. We recorded the ventilator settings of $\mathrm{F}_{\mathrm{I}} \mathrm{O}_{2}$ and mean airway pressure (MAP) and calculated the $\mathrm{F}_{\mathrm{I}} \mathrm{O}_{2} \times \mathrm{MAP}$ as a marker of invasive ventilation.

\section{Histological evaluation and immunohistochemistry}

Formalin-fixed paraffin-embedded tissue blocks of lung specimens were retrieved from the autopsy of Hiroshima University Hospital (Hiroshima, Japan). Numerous microsections stained with hematoxylin and eosin, Massontrichrome, and elastica van Gieson were reviewed by two pathologists (VJ Amatya and Y Takeshima). Fibrosis was evaluated in Masson-trichrome-stained microsections.

Immunohistochemistry of the biomarkers, except KL6 , was conducted using microsections prepared from the patients' best representative formalin-fixed, paraffinembedded blocks. Immunohistochemical staining was conducted using an automated immunohistochemical station (Benchmark GX; Ventana-Roche, Tokyo, Japan) with the Ultraview Universal DAB Detection Kit (VentanaRoche, Los Angeles, CA, USA). The antigen was retrieved by using the Cell Conditioning 1 solution (VentanaRoche, Los Angeles, CA, USA). The antibodies used in this study were SP-D (Bioss Antibodies, Woburn, MA, USA), angiopoietin 2 (R\&D Systems, Minneapolis, MN, USA), receptor for advanced glycation end products (RAGE) (R\&D), IL-6 (R\&D), and IL-8 (Proteintech Group, Rosemont, IL, USA). The microsection was counterstained with Mayer's hematoxylin.

For immunohistochemistry of anti-KL-6, the antiKL-6 mouse IgG1 monoclonal antibody (mAb), purified using a protein A affinity column (Affi Gel Protein A MAPS II Kit; Bio-Rad, Hercules, CA, USA) from the ascites collected from mice bearing anti-KL-6 mAb- producing hybridomas as previously described (22), was used. The slides were immersed in Target Retrieval Solution, Citrate pH 6 (Dako Japan, Tokyo, Japan) and boiled at $108{ }^{\circ} \mathrm{C}$ for 15 minutes in an autoclave for antigen retrieval. After blocking endogenous peroxidase activity with $0.03 \%$ hydrogen peroxide for 30 minutes, a mouse anti-human KL-6 mAb was added to the sections. Sections were incubated with a secondary antibody, horseradish peroxidase-labeled antimouse immunoglobulin G (IgG), followed by the addition of a substrate-chromogen. It was then counterstained with Mayer's hematoxylin.

Immunohistochemical evaluation was independently semi-quantified by VJA and YK. A "positive case" was defined as immunoreactive expression on $>20 \%$ of alveolar cells and a "strong positive case" was defined as immunoreactive expression on $>50 \%$ of alveolar cells. For the histochemical assessment of the specimens, the pathologists were blinded to the clinical course and treatments.

\section{Statistical analysis}

All data are expressed as the median and interquartile range. All statistical analyses were conducted using SPSS version 23.0 for Mac (SPSS Inc., Chicago, IL, USA). Differences were statistically significant at $\mathrm{P}<0.05$. Nonnormally distributed variables between the two groups were compared by using the Mann-Whitney $U$ test. Categorical variables between the two groups were compared by using Fisher's exact probability and the chi-square test.

\section{Results}

\section{Patients' characteristics}

The patients' ages and sex were similar between the ventilator and ECMO groups (median age, 65 vs. 64 years; $\mathrm{P}=1.00)$. APACHE II scores were significantly different between the two groups (14.5 vs. 35.0; $\mathrm{P}=0.006)$. Serum KL-6 levels at the time of admission into the ICU, at 7 days after ICU admission, and at 14 days after ICU admission were not significantly different between the two groups. The ventilator days were significantly shorter in the ventilator group than in the ECMO group (17.5 vs. 30.0 days; $\mathrm{P}=0.04)$. The duration of ICU stay was also shorter in the ventilator group than in the ECMO group (17.5 vs. 30 days; $\mathrm{P}=0.02)$. The $\mathrm{F}_{\mathrm{I}} \mathrm{O}_{2} \times \mathrm{MAP}$ values on day 0 , day 7 , and day 14 tended to be lower in the ECMO group than in the 
Table 1 Clinical characteristics of the patients

\begin{tabular}{lccc}
\hline Indexes & Ventilator group $(\mathrm{n}=6)$ & ECMO group $(\mathrm{n}=9)$ & $\mathrm{P}$ value \\
\hline Age $(\mathrm{y})$ & $65[62-68]$ & $64[61-69]$ & 1.00 \\
Men/women & $5 / 1$ & $7 / 2$ & \\
APACHEII & $14.5[12.3-17.5]$ & $35.0[28.0-36.0]$ & 0.006 \\
score & & & \\
SOFA score & $4.0[3.25-5.5]$ & $11.0[8.0-11.0]$ & 0.005 \\
Serum & & & \\
KL-6 (U/L) & & & \\
Day 0 & $1,482[771-2,339]$ & $1,301[484-2,263]$ & 0.53 \\
Day 7 & $1,559[1,460-2,339]$ & $1,197[459-1,536]$ & 0.22 \\
Day 14 & $1,810[1,572-2,731]$ & $1,416[484-2,263]$ & 0.22 \\
F,O $\times$ MAP & & & \\
Day 0 & $12.3[10.9-17.6]$ & $4.8[3.6-5.2]$ & 0.01 \\
Day 7 & $5.6[4.8-11]$ & $8.4[5.0-9.3]$ & 0.76 \\
Day 14 & $13.7[9.3-18.0]$ & $5.2[3.8-9.5]$ & 0.12 \\
Length of & $17.5[14-19]$ & $30.0[24-41]$ & 0.02 \\
ICU stay (d) & & & \\
Duration of & $17.5[11-18]$ & $30.0[24-41]$ & 0.04 \\
mechanical & & & \\
ventilation $(d)$ & & & \\
Duration of & & & \\
ECMO $(d)$ & & & \\
\hline
\end{tabular}

The values are expressed as the median [interquartile range]. APACHE, Acute Physiology and Chronic Health Evaluation; $\mathrm{ECMO}$, extracorporeal membrane oxygenation; $\mathrm{F}_{1} \mathrm{O} 2$, fraction of inspired oxygen; ICU, intensive care unit; KL-6, Krebs von den Lungen-6; MAP, mean airway pressure.

ventilator group ( 4.8 vs. $12.3, \mathrm{P}=0.01 ; 8.4$ vs. $5.6, \mathrm{P}=0.76$; and 5.2 vs. 13.7, $\mathrm{P}=0.12$, respectively) (Table 1).

\section{Histological and immunobistological findings}

The results of the histological and immunohistological studies are summarized in Table 2. Fibrosis (Massontrichrome stain) and KL-6 and IL-8 immunoreactivity were stronger in the ventilator group than in the ECMO group (Figure 1 and Table 2). Positive staining for SP-D showed a decreased trend in the ECMO group (Table 2).

In both groups, type II alveolar epithelial cells exhibited inflammatory proteins (IL-6 and IL-8) and epithelial and angiogenetic proteins (Ang2 and RAGE) (Figures 2 and 3). In addition, IL-8 tended to be expressed in fibroblasts.

\section{Discussion}

This study showed that positive staining of index of fibrosis with Masson's trichrome stain and the IP marker of KL6 , were stronger in the ventilator group compared with the ECMO group, despite the longer ventilator days in the ECMO group. In addition, IL-8 was less frequently expressed in the ECMO group than that in the ventilator group. These data suggest that the management using $\mathrm{V}-\mathrm{V}$ ECMO for AE-IP patients might have suppressed fibrosis, inflammation and alveolar epithelial damage.

The fibrosis marker KL-6 was strongly expressed on type II alveolar pneumocytes and bronchiolar epithelial cells in patients diagnosed as having IP (23). Surfactant protein D has been reported as a useful biomarker for AE-IP (24). The expression of SP-D is evidenced on alveolar type II cells and the Clara cells (25). Inflammation of the lungs is promoted by IL-8, which indirectly triggers fibrosis. The decreased release of IL-8 from alveolar epithelial type II cells and macrophages due to alleviating lung stretch by diminishing ventilatory stress could have caused lung inflammation and subsequent fibrosis in the ECMO group $(26,27)$.

No significant difference existed in the positive staining ratio of Ang2 and RAGE between the ECMO group and the ventilator group. Angiopoietin 2 is an endothelial growth factor and regulates vascular permeability (28). The serum Ang2 level reflects pulmonary damage and pulmonary inflammation in patients with AE-IP (29). In addition, RAGE is expressed on the basolateral membrane of alveolar type I and II epithelial cells in the lung (30). It has been implicated in the lung fibrotic process and in alveolar homeostasis (31). Based on our findings, a tendency for the suppression of RAGE in the ECMO group suggested the involvement of endothelial injury and inflammation. The exact roles and function of these markers remain to be explored (32).

The mortality rate for patients with $\mathrm{AE}-\mathrm{IP}$ receiving mechanical ventilation is extremely high (6). The reasons for the high mortality may be the rapid progressive nature of diffuse alveolar damage, which may be partly caused by mechanical ventilation. In this study, lung protective ventilator settings, evaluated by using the $\mathrm{F}_{\mathrm{I}} \mathrm{O}_{2}$ /MAP ratio, tended to be lower in the ECMO group. This finding may suggest the contribution of $\mathrm{V}-\mathrm{V}$ ECMO support in decreasing the ventilator settings and subsequently decreasing lung injury.

Our study has several limitations. First, this study was retrospective and included a limited number of patients, 
Table 2 Positive ratio of immunohistological staining

\begin{tabular}{|c|c|c|c|c|c|}
\hline Stain & \multicolumn{2}{|c|}{ Ventilator group $(n=6)$} & \multicolumn{2}{|c|}{ ECMO group $(n=9)$} & $P$ value \\
\hline Masson-trichrome & $0[0]$ & $6[100]$ & $5[56]$ & $4[44]$ & 0.04 \\
\hline SP-D & $0[0]$ & $6[100]$ & $4[44]$ & $5[56]$ & 0.10 \\
\hline KL-6 & $0[0]$ & $6[100]$ & $5[56]$ & $4[44]$ & 0.04 \\
\hline IL-8 & $0[0]$ & $6[100]$ & $6[67]$ & 3 [23] & 0.02 \\
\hline Ang 2 & $2[33]$ & $4[67]$ & 8 [89] & $1[11]$ & 0.08 \\
\hline RAGE & $4[67]$ & $2[33]$ & 8 [89] & $1[11]$ & 0.52 \\
\hline
\end{tabular}

$\mathrm{a}$, for the comparison between the strong positive groups, the $\mathrm{P}$ values are based on the Fisher exact test; $\mathrm{b}$, the data are based on a total of four samples. Ang2, angiopoietin 2; ECMO, extracorporeal membrane oxygenation; IL-6, interleukin 6; IL-8, interleukin 8; KL-6, Krebs von den Lungen-6; RAGE, receptor for advanced glycosylation end products; SOFA, Sequential Organ Failure Assessment; SP-D, pulmonary surfactant protein $\mathrm{D}$.
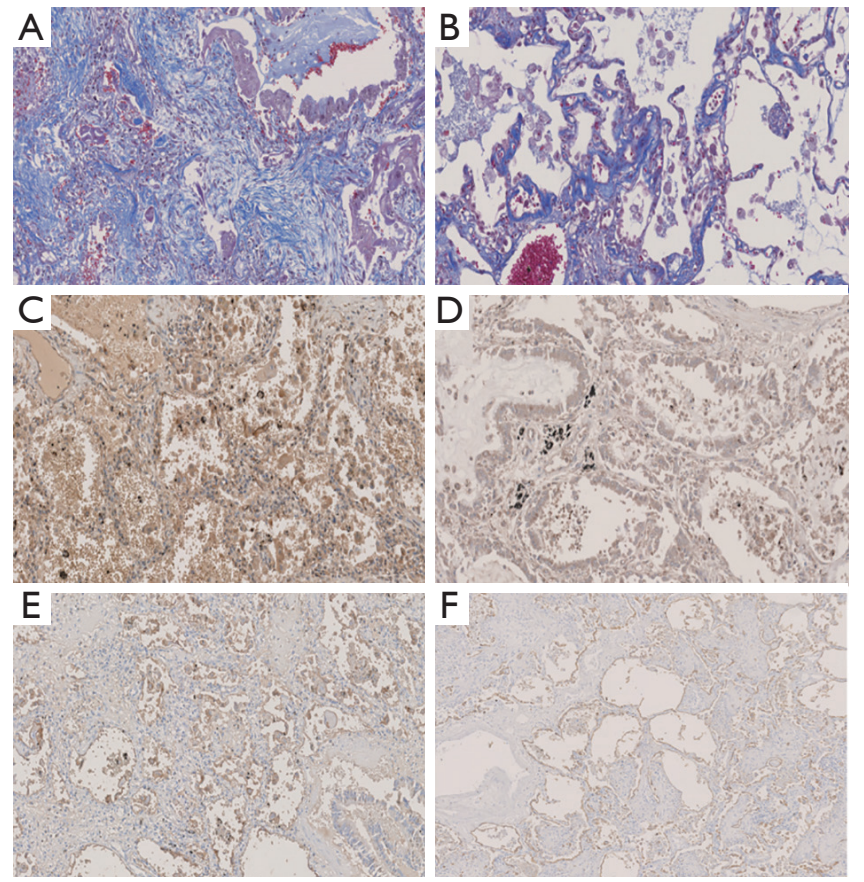

Figure 1 Immunohistological images of autopsied lung specimens. Masson-trichrome staining is stronger in the ventilator group (A) than in the ECMO group (B) (magnification, 400x). Pulmonary surfactant protein $\mathrm{D}$ immunoreactivity is stronger in the ventilator group (C) than in the ECMO group (D) (magnification, 400x). Krebs von den Lungen-6 immunoreactivity is stronger in the ventilator group (E) than in the ECMO group (F) (magnification, 400x). ECMO, extracorporeal membrane oxygenation.
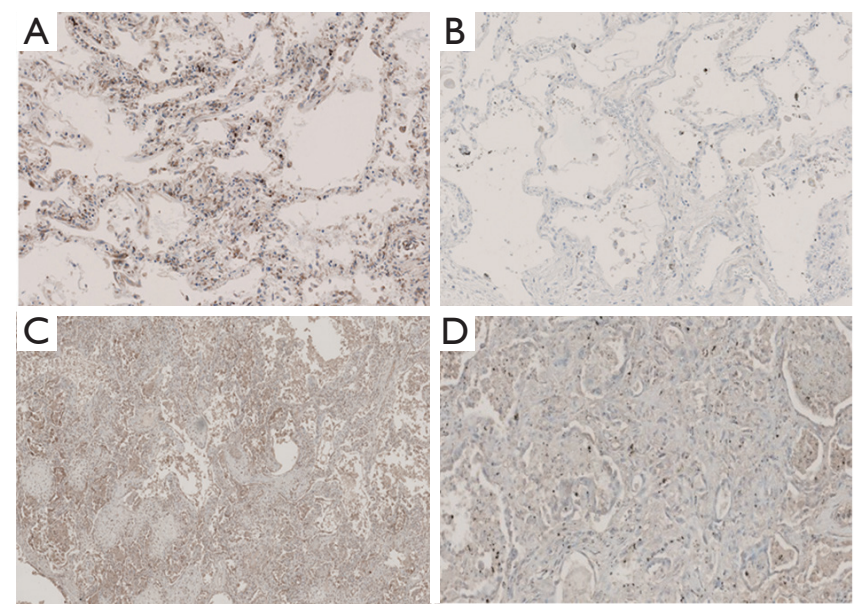

Figure 2 Immunohistological images of autopsied lung specimens. IL-6 immunoreactivity is not significantly difference between in the ventilator group (A) and in the ECMO group (B) (magnification, 400x). IL-8 immunoreactivity is stronger in the ventilator group $(\mathrm{C})$ than in the ECMO group (D) (magnification, $400 \times$ ). ECMO, extracorporeal membrane oxygenation; IL-6, interleukin 6; IL-8, interleukin 8.

which may cause a beta-error. The limited number of patients is because of the scarcity of the disease itself and the difficulty in obtaining consent for an autopsy. Second, patient characteristics between the two groups were not comparable because patients with cases of more severe 

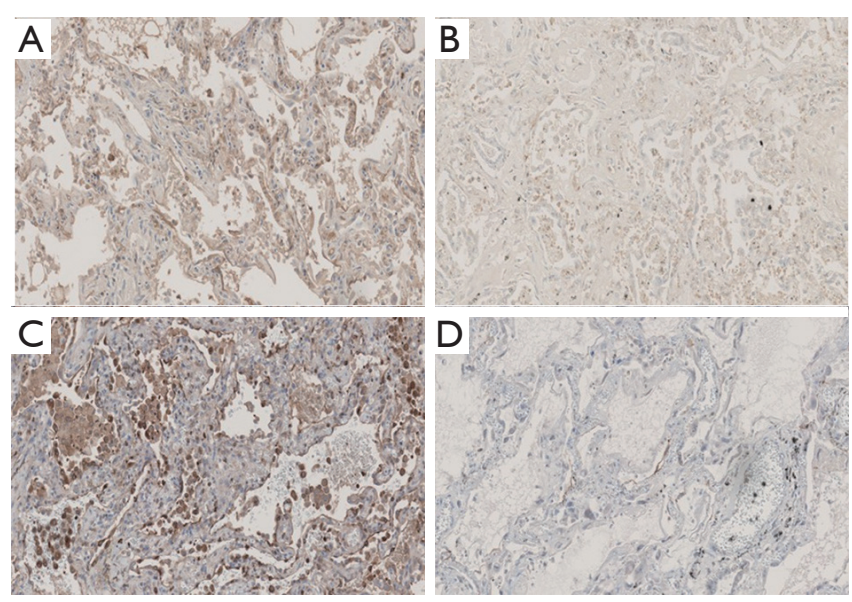

Figure 3 Immunohistological images of autopsied lung specimens. Ang2 immunoreactivity is not significantly differences between in the ventilator group (A) and in the ECMO group (B) (magnification, 400x). RAGE immunoreactivity is not significantly difference between in the ventilator group (C) and in the ECMO group (D) (magnification, 400x). Ang2, angiopoietin 2; ECMO, extracorporeal membrane oxygenation; RAGE, receptor for advanced glycosylation end products.

pulmonary cases tended to have ECMO therapy. Third, since the data on histological examination of patients before mechanical ventilation in both the groups was not available, the changes during the treatment period were not able to be examined. Thus, because of the nature of the study did not allow the study of the influence of several important factors that might have affected lung tissue (i.e., immunosuppressive drugs and bacterial, viral and fungal infections) were not studied. Fourthly, the positive rate of antibody cannot be quantified by qualitative evaluation alone in a histopathological examination. Fifthly, validation is necessary in a biopsy to assess the relevance of evaluating the histological findings.

\section{Conclusions}

The immunohistochemical and histological evaluations of autopsied lungs from patients with AE-IP suggested that the application of $\mathrm{V}-\mathrm{V}$ ECMO may be associated with alleviating alveolar epithelial injury, inflammation, and collagen synthesis in the lungs. Further studies would need to reveal the detail of mechanism of progression of fibrosis in AE-IP patients and its attenuating effects of lung protective management.

\section{Acknowledgments}

The authors wish to acknowledge Dr. Kakuhiro Yamaguchi (University of Hiroshima, Hiroshima, Japan) for his assistance in the immunohistochemical evaluations.

Funding: This work was supported by the Japan Society for the Promotion of Science (JSPS; Tokyo, Japan) KAKENHI (grant No. JP17K17052).

\section{Footnote}

Conflicts of Interest: The authors have no conflicts of interest to declare.

Ethical Statement: The authors are accountable for all aspects of the work in ensuring that questions related to the accuracy or integrity of any part of the work are appropriately investigated and resolved. The experiments in this study comply with the current laws of Japan, and were approved by the ethics committee in our institution (Hiroshima University, Hiroshima, Japan; project approval No.: RIN-231).

\section{References}

1. Kondoh $\mathrm{Y}$, Taniguchi H, Kawabata $\mathrm{Y}$, et al. Acute exacerbation in idiopathic pulmonary fibrosis. Analysis of clinical and pathologic findings in three cases. Chest 1993;103:1808-12.

2. Munshi L, Walkey A, Goligher E, et al. Venovenous extracorporeal membrane oxygenation for acute respiratory distress syndrome: a systematic review and meta-analysis. Lancet Respir Med 2019;7:163-72.

3. Kim DS, Park JH, Park BK, et al. Acute exacerbation of idiopathic pulmonary fibrosis: frequency and clinical features. Eur Respir J 2006;27:143-50.

4. Richeldi L, Davies HR, Ferrara G, et al. Corticosteroids for idiopathic pulmonary fibrosis. Cochrane Database Syst Rev 2003;(3):CD002880.

5. Davies HR, Richeldi L, Walters EH. Immunomodulatory agents for idiopathic pulmonary fibrosis. Cochrane Database Syst Rev 2003;(3):CD003134.

6. Mallick S. Outcome of patients with idiopathic pulmonary fibrosis (IPF) ventilated in intensive care unit. Respir Med 2008;102:1355-9.

7. Gaudry S, Vincent F, Rabbat A, et al. Invasive mechanical ventilation in patients with fibrosing interstitial pneumonia. J Thorac Cardiovasc Surg 2014;147:47-53. 
8. Peek GJ, Mugford M, Tiruvoipati R, et al. Efficacy and economic assessment of conventional ventilatory support versus extracorporeal membrane oxygenation for severe adult respiratory failure (CESAR): a multicentre randomised controlled trial. Lancet 2009;374:1351-63.

9. International consensus conferences in intensive care medicine: Ventilator-associated Lung Injury in ARDS. This official conference report was cosponsored by the American Thoracic Society, The European Society of Intensive Care Medicine, and The Societe de Reanimation de Langue Francaise, and was approved by the ATS Board of Directors, July 1999. Am J Respir Crit Care Med 1999; 160:2118-24.

10. Brodie D, Bacchetta M. Extracorporeal membrane oxygenation for ARDS in adults. N Engl J Med 2011;365:1905-14.

11. Acute Respiratory Distress Syndrome Network, Brower RG, Matthay MA, et al. Ventilation with lower tidal volumes as compared with traditional tidal volumes for acute lung injury and the acute respiratory distress syndrome. N Engl J Med 2000;342:1301-8.

12. Australia and New Zealand Extracorporeal Membrane Oxygenation (ANZ ECMO) Influenza Investigators, Davies A, Jones D, et al. Extracorporeal Membrane Oxygenation for 2009 Influenza A(H1N1) Acute Respiratory Distress Syndrome. JAMA 2009;302:1888-95.

13. Calfee CS, Janz DR, Bernard GR, et al. Distinct Molecular Phenotypes of Direct vs Indirect ARDS in Single-Center and Multicenter Studies. Chest 2015;147:1539-48.

14. Thille AW, Esteban A, Fernandez-Segoviano P, et al. Comparison of the Berlin definition for acute respiratory distress syndrome with autopsy. Am J Respir Crit Care Med 2013;187:761-7.

15. Mukhopadhyay S, Parambil JG. Acute interstitial pneumonia (AIP): relationship to Hamman-Rich syndrome, diffuse alveolar damage (DAD), and acute respiratory distress syndrome (ARDS). Semin Respir Crit Care Med 2012;33:476-85.

16. American Thoracic Society; European Respiratory Society. American Thoracic Society/European Respiratory Society International Multidisciplinary Consensus Classification of the Idiopathic Interstitial Pneumonias. This joint statement of the American Thoracic Society (ATS), and the European Respiratory Society (ERS) was adopted by the ATS board of directors, June 2001 and by the ERS Executive Committee, June 2001. Am J Respir Crit Care Med 2002;165:277-304.

17. Katzenstein AL, Bloor CM, Leibow AA. Diffuse alveolar damage--the role of oxygen, shock, and related factors. A review. Am J Pathol 1976;85:209-28.

18. Ashbaugh DG, Bigelow DB, Petty TL, et al. Acute respiratory distress in adults. Lancet 1967;2:319-23.

19. Collard HR, Ryerson CJ, Corte TJ, et al. Acute Exacerbation of Idiopathic Pulmonary Fibrosis. An International Working Group Report. Am J Respir Crit Care Med 2016;194:265-75.

20. Bjoraker JA, Ryu JH, Edwin MK, et al. Prognostic significance of histopathologic subsets in idiopathic pulmonary fibrosis. Am J Respir Crit Care Med 1998;157:199-203.

21. Flaherty KR, Thwaite EL, Kazerooni EA, et al. Radiological versus histological diagnosis in UIP and NSIP: survival implications. Thorax 2003;58:143-8.

22. Kohno N, Akiyama M, Kyoizumi S, et al. Detection of soluble tumor-associated antigens in sera and effusions using novel monoclonal antibodies, KL-3 and KL6, against lung adenocarcinoma. Jpn J Clin Oncol 1988;18:203-16.

23. Kohno N, Kyoizumi S, Awaya Y, et al. New serum indicator of interstitial pneumonitis activity. Sialylated carbohydrate antigen KL-6. Chest 1989;96:68-73.

24. Ohnishi H, Yokoyama A, Kondo K, et al. Comparative study of KL-6, surfactant protein-A, surfactant protein-D, and monocyte chemoattractant protein- 1 as serum markers for interstitial lung diseases. Am J Respir Crit Care Med 2002;165:378-81.

25. Takahashi H, Fujishima T, Koba H, et al. Serum surfactant proteins $\mathrm{A}$ and $\mathrm{D}$ as prognostic factors in idiopathic pulmonary fibrosis and their relationship to disease extent. Am J Respir Crit Care Med 2000;162:1109-14.

26. Ware LB, Koyama T, Zhao Z, et al. Biomarkers of lung epithelial injury and inflammation distinguish severe sepsis patients with acute respiratory distress syndrome. Crit Care 2013;17:R253.

27. Mascarenhas MM, Day RM, Ochoa CD, et al. Low molecular weight hyaluronan from stretched lung enhances interleukin-8 expression. Am J Respir Cell Mol Biol 2004;30:51-60.

28. Agrawal A, Matthay MA, Kangelaris KN, et al. Plasma angiopoietin-2 predicts the onset of acute lung injury in critically ill patients. Am J Respir Crit Care Med 2013;187:736-42.

29. Ando M, Miyazaki E, Abe T, et al. Angiopoietin-2 expression in patients with an acute exacerbation of idiopathic interstitial pneumonias. Respir Med 2016;117:27-32. 
30. Katsuoka F, Kawakami Y, Arai T, et al. Type II alveolar epithelial cells in lung express receptor for advanced glycation end products (RAGE) gene. Biochem Biophys Res Commun 1997;238:512-6.

31. Machahua C, Montes-Worboys A, Llatjos R, et al.

Cite this article as: Kida Y, Ohshimo S, Kyo M, Hosokawa K, Amatya VJ, Takeshima Y, Shime N. Retrospective immunohistological study of autopsied lungs in patients with acute exacerbation of interstitial pneumonia managed with extracorporeal membrane oxygenation. J Thorac Dis 2019;11(11):4436-4443. doi: 10.21037/jtd.2019.11.09
Increased AGE-RAGE ratio in idiopathic pulmonary fibrosis. Respir Res 2016;17:144.

32. Chuah YK, Basir R, Talib H, et al. Receptor for advanced glycation end products and its involvement in inflammatory diseases. Int J Inflam 2013;2013:403460. 